\title{
A Parametric Computational Study of Boron Transport in Hall Thrusters
}

\author{
Brandon D. Smith* and Iain D. Boyd ${ }^{\dagger}$ \\ Nonequilibrium Gas and Plasma Dynamics Laboratory \\ University of Michigan, Ann Arbor, Michigan, 48109, USA
}

\begin{abstract}
The hybrid fluid/particle-in-cell model HPHall is applied to simulate the transport of sputtered boron atoms through NASA's HiVHAc Hall thruster. The dependence of the spatial distribution of boron on the velocity distribution functions (VDFs) used to initialize boron macroparticles is investigated. Assuming a Maxwell-Boltzmann distribution for the forward and transverse sputtering directions, the bulk velocity and temperature of the sputtered atoms are varied, and the resulting boron density profiles are analyzed and compared to experimental measurements. The bulk velocity of the sputtered atoms largely controls the amount of boron that escapes into the thruster plume, with larger bulk velocities resulting in a greater boron density, whereas the temperature of the sputtered atoms determines how boron is distributed radially in the plume. At a higher temperature, the qualitative radial distribution of boron predicted by the numerical model more closely resembled that of experimental measurements taken using cavity ring-down spectroscopy. Ultimately, both the bulk velocity and the temperature of the sputtered atoms have a significant effect on the distribution of boron atoms in the thruster plume, so the dependence of both of these parameters on incident ion properties must be considered in future studies.
\end{abstract}

\section{Introduction}

$\mathrm{T}$ HE erosion of the discharge channel walls in Hall thrusters is a fundamental life-limiting process that also produces free condensible material that may deposit on thruster and spacecraft surfaces. This erosion process occurs over a long period of time - on the order of 10,000 hours for complete erosion of the wallsmaking experimental characterization of the process both time-consuming and costly. Hence, it is desirable to apply numerical modeling in an effort to describe wall erosion and estimate the operational lifetime of Hall thrusters.

The present work seeks to build on a previous effort that applied the hybrid fluid/particle-in-cell (hybridPIC) model HPHall ${ }^{1}$ to predict the transport of boron atoms produced by wall erosion through a Hall thruster discharge channel. ${ }^{2,3}$ The previous work utilized results from a high-speed molecular dynamics (MD) model, ${ }^{3-6}$ including total sputter yields and sputtered boron velocity distribution functions (VDFs), to dynamically calculate the erosion rate of the thruster walls and introduce boron particles into the simulation domain. However, contrary to the findings of the MD model, the boron VDF was assumed to be independent of the kinetic energy and incidence angle of the bombarding ions. In principle, the 3D VDF affects how the boron atoms propagate through the plasma, and in turn determines the spatial distribution of boron atoms in the discharge channel and plume. Although the MD model predicts that the 3D VDF is dependent on the properties of the incident ions, it is not obvious how this dependence influences boron density profiles in a real thruster. Hence, this work utilizes a parametric analysis to study the sensitivity of boron density profiles in the near-field plume of a Hall thruster to the VDF parameters used to initialize the boron particles.

The remainder of this paper is organized as follows: Section II describes the simulation methodology, including the numerical model used, the thruster modeled, and the input parameters. Section III presents the simulation results and compares them to experimental measurements. Finally, Section IV summarizes the findings and discusses possibilities for future studies.

\footnotetext{
*Postdoctoral Research Fellow, Department of Aerospace Engineering, AIAA Member.

$\dagger$ James E. Knott Professor, Department of Aerospace Engineering, AIAA Fellow.
} 


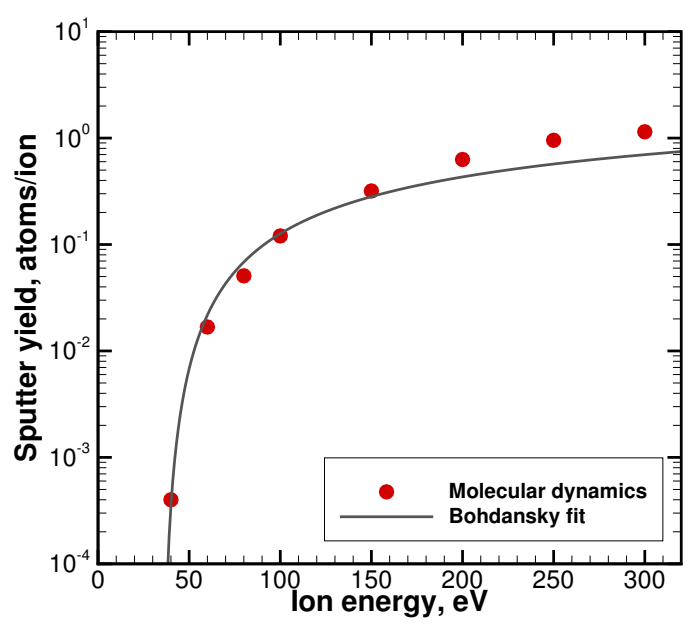

(a) Energy dependence at $0^{\circ}$ incidence

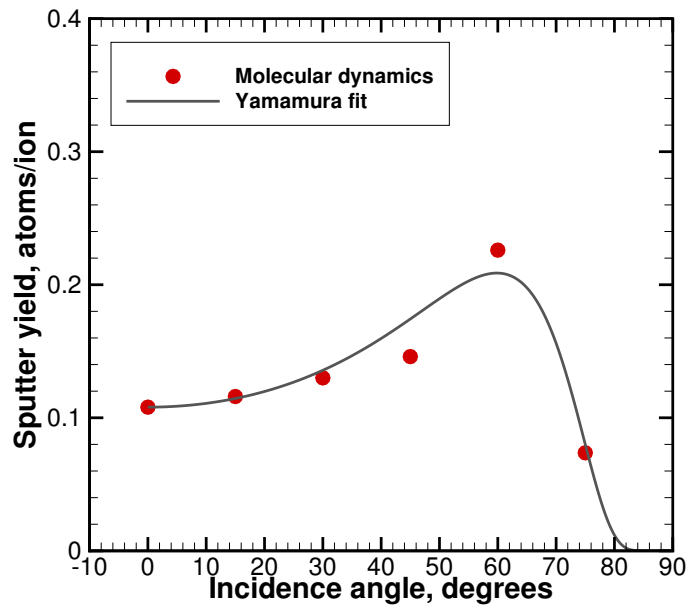

(b) Angular dependence at $100 \mathrm{eV}$ energy

Figure 1: Energy and angular dependence of the total sputter yield of $h$-BN as calculated from the MD model. Fitted curves are implemented in HPHall-3.

\section{Simulation Methodology}

\section{A. Numerical Model}

The numerical model used in this work is HPHall, a hybrid code for simulating a Hall thruster plasma discharge. ${ }^{1}$ HPHall models electrons using a quasi-1D fluid approach wherein the Boltzmann relation is solved along magnetic field lines and a generalized Ohm's law is solved across magnetic field lines. The heavy species are treated using a 2D axisymmetric particle-in-cell (PIC) method. The electron and heavy species submodels are linked through the assumption of quasineutrality, and the plasma density is determined from the heavy species submodel. The most recent version of HPHall, called HPHall-3, is used for this work..$^{7,8}$

Through past efforts, several steps were taken to make HPHall-3 capable of modeling the transport of erosion products in Hall thrusters. First, a high-speed, high-fidelity molecular dynamics (MD) model ${ }^{3-6}$ was utilized to investigate the sputtering of hexagonal boron nitride $(h-\mathrm{BN})$ under xenon ion bombardment. The total sputter yields of $h$-BN were calculated and the properties of the sputtered particles were analyzed, revealing that that $h$-BN sputters primarily in the form of B and $\mathrm{N}_{2}$. Second, semi-empirical curve fits were applied to the calculated sputter yields and implemented in HPHall-3, allowing dynamic calculation of the wall erosion rate as a simulation progresses. Finally, velocity distribution functions obtained from the MD analysis for atomic boron, the most abundant condensible product of erosion, were included in HPHall-3 to provide initial conditions for boron macroparticles introduced at the walls where erosion occurs.

The total sputter yield calculated from the MD model is shown in Fig. 1 as a function of ion energy at normal $\left(0^{\circ}\right)$ incidence and as a function of ion incidence angle at $100 \mathrm{eV}$ energy. For reference, the energy distribution function of ions striking the wall is shown in Fig. 2 for a thruster operating at $400 \mathrm{~V}$. The sputter yield as a function of ion energy is fitted in a least-squares sense using a semi-empirical Bohdansky function: ${ }^{9}$

$$
Y_{B}\left(\mathcal{E}_{i}\right)=\alpha s_{n}(\epsilon)\left[1-\left(\frac{E_{t h}}{\mathcal{E}_{i}}\right)^{2 / 3}\right]\left(1-\frac{E_{t h}}{\mathcal{E}_{i}}\right)^{2} .
$$

where $s_{n}(\epsilon)$ is the nuclear stopping cross-section and $\alpha$ and $E_{t h}$ are treated as free fit parameters. Physically, $E_{t h}$ is interpreted as the threshold energy for sputtering. In the previous work, $s_{n}$ was assumed to be constant with ion energy and was given a value of 1 for simplicity. In this work, the nuclear stopping cross-section is given by

$$
s_{n}(\epsilon)=\frac{0.5 \ln (1+1.2288 \epsilon)}{\epsilon+0.1728 \sqrt{\epsilon}+0.008 \epsilon^{0.1504}},
$$


where $\epsilon$ is the reduced ion energy:

$$
\begin{aligned}
\epsilon & =\mathcal{E}_{i} \frac{m_{t}}{m_{i}+m_{t}} \frac{a_{L}}{Z_{i} Z_{t} e^{2}}, \\
a_{L} & \approx \frac{0.8853 a_{0}}{\left(Z_{i}^{2 / 3}+Z_{t}^{2 / 3}\right)^{1 / 2}} .
\end{aligned}
$$

This form was proposed by García-Rosales et al. based on a derivation from a Krypton-Carbon potential function, ${ }^{10}$ which is very similar to the Ziegler-Biersack-Littmark potential used in the MD sputtering model. ${ }^{11,12}$ The least-squares fit of the Bohdansky function to the calculated sputter yields at normal ion incidence results in these values for the free fit parameters: $\alpha=23$ and $E_{t h}=35 \mathrm{eV}$. These are the values used in HPHall-3.

The sputter yield as a function of ion incidence angle is fitted using a Yamamura function: ${ }^{13}$

$$
\hat{Y}_{Y}\left(\theta_{i}\right)=\frac{Y_{Y}\left(\theta_{i}\right)}{Y_{0}}=\cos ^{A}\left(\theta_{i}\right) \exp \left[-B\left(\frac{1}{\cos \left(\theta_{i}\right)}-1\right)\right]\left[\frac{1-\sqrt{\frac{E^{*}}{\mathcal{E}_{i}}} \cos \left(\theta_{i}\right)}{1-\sqrt{\frac{E^{*}}{\mathcal{E}_{i}}}}\right] .
$$

where $Y_{0}$ is the yield at normal incidence and $A$, $B$, and $E^{*}$ are free fit parameters. The values used in this work are $A=-3.5, B=1.7$, and $E^{*}=0$. These values correspond to an incident ion energy of $100 \mathrm{eV}$, although similar values are found at other ion energies. ${ }^{3,5,6}$

The Bohdansky and Yamamura curve fits are implemented within HPHall-3 and are used to calculate the sputter yield whenever an ion macroparticle strikes a wall according to:

$$
Y\left(\mathcal{E}_{i}, \theta_{i}\right)=Y_{B}\left(\mathcal{E}_{i}\right) \hat{Y}_{Y}\left(\theta_{i}\right)
$$

For each sputtering event, a number of boron macroparticles are introduced into the simulation domain such that the total number of real atoms corresponds to the calculated sputter yield. The initial velocity of each macroparticle comes from VDFs fitted to the MD data. ${ }^{5}$ The VDF in the wall-normal direction is the Sigmund-Thompson distribution: ${ }^{14}$

$$
f\left(v_{n}\right) \propto \frac{v_{n}^{2}}{\left(v_{n}^{2}+v_{b}^{2}\right)^{3-2 m}}
$$

where $m$ and $v_{b}$ are treated as free fit parameters. The quantity $v_{b}$ is the effective binding velocity which is related to the surface binding energy $U_{b}$ as

$$
U_{b}=\frac{1}{2} m_{B} v_{b}^{2}
$$

In the linear cascade regime, $U_{b}$ is independent of the incident ion properties, indicating that it is a property of the material. ${ }^{14}$ The MD data indicate that the binding energy is approximately constant for ion energies above $100 \mathrm{eV}$, taking an average value of $U_{b}=4.5 \mathrm{eV} .^{3,5,6}$ The quantity $m$ varies between 0 and $\sim 0.3$, but is zero for most MD simulation cases. Hence, the Sigmund-Thompson distribution is considered independent of the incident ion's properties in the present work, as was the case in the previous work. ${ }^{2,3}$

The VDFs along the dimensions parallel to the walls (called the forward and transverse sputtering directions) are also determined from the MD data, and are reasonably well described by a Maxwell-Boltzmann distribution. The MD data indicate that the temperature of the sputtered boron atoms has a strong dependence on the incident ion's energy and angle of incidence, and that the mean velocity in the forward 


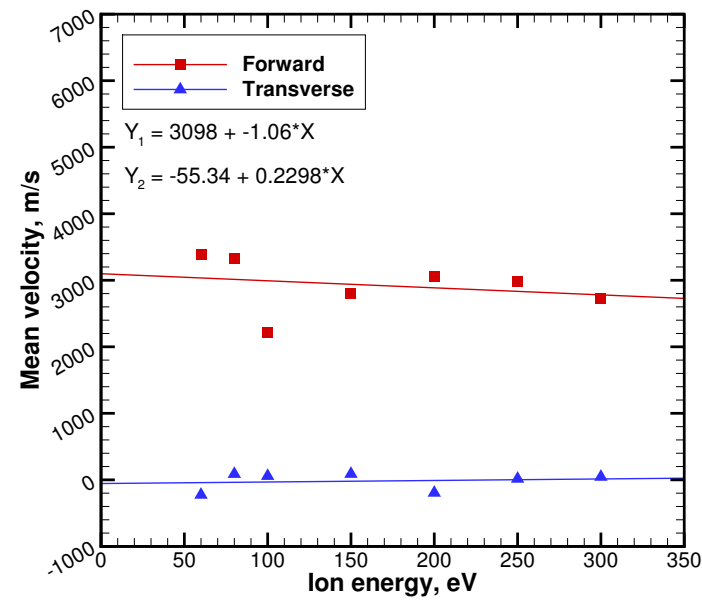

(a) Mean velocity

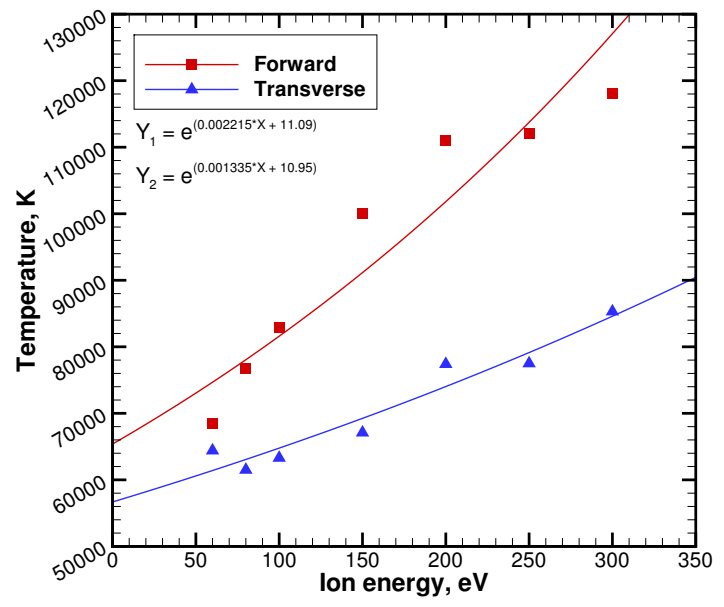

(b) Temperature

Figure 3: Mean velocity and temperature of sputtered boron atoms as a function of incident ion energy for $60^{\circ}$ incidence. Data are fitted with linear or exponential functions for reference.

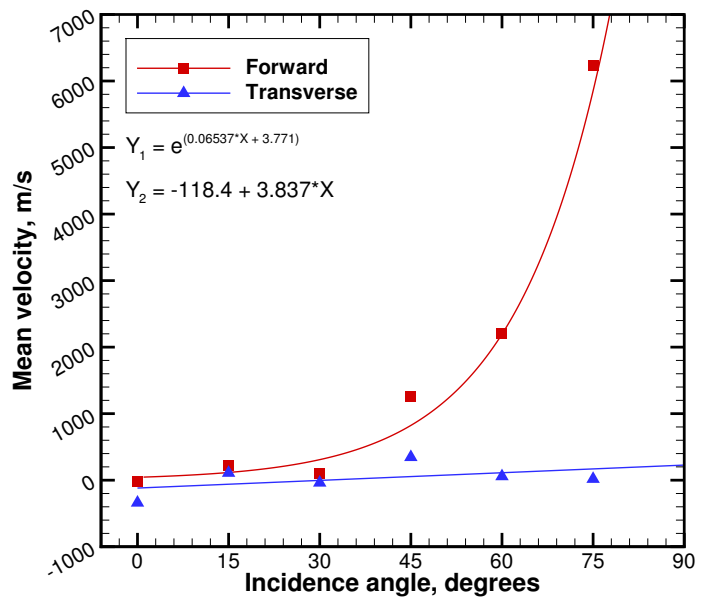

(a) Mean velocity

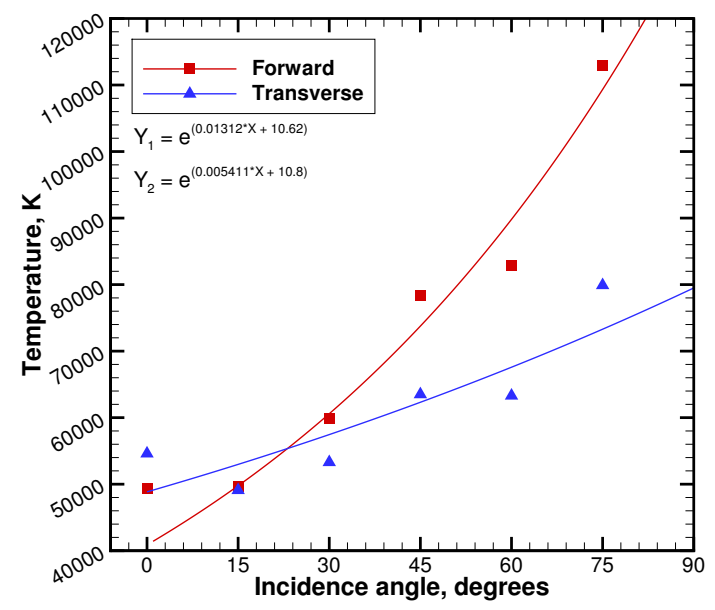

(b) Temperature

Figure 4: Mean velocity and temperature of sputtered boron atoms as a function of ion incidence angle for $100 \mathrm{eV}$ energy. Data are fitted with linear or exponential functions for reference. 


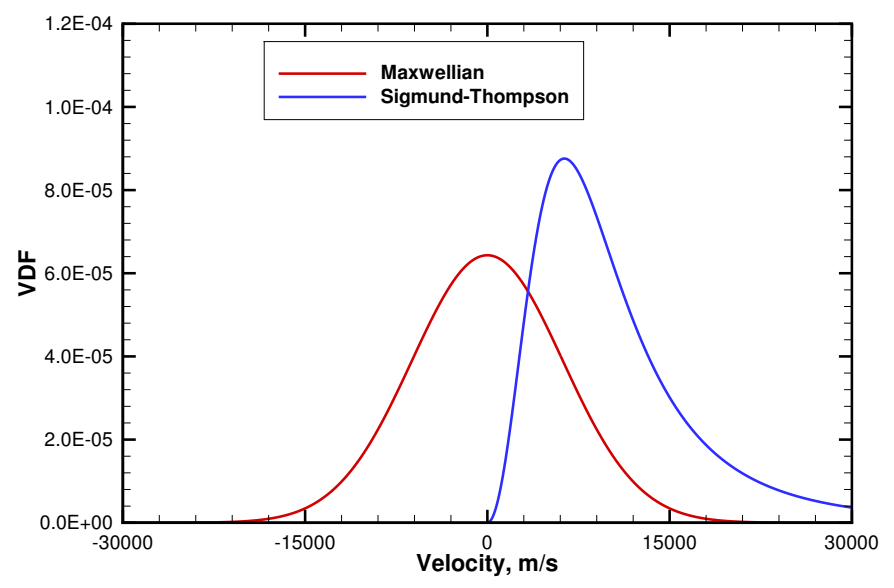

Figure 5: Sigmund-Thompson and Maxwell-Boltzmann velocity distribution functions previously used to initialize boron macroparticles in HPHall-3.

sputtering direction has a strong dependence primarily on the ion incidence angle., ${ }^{3,5,6}$ This is shown in Figures 3 and ??.

In principle, variation in the Maxwellian bulk velocity and temperature with ion properties may have significant effects on the the spatial distribution of boron in a Hall thruster discharge channel and plume. However, for the sake of simplicity, the previous work assumed constant values of zero for the mean velocity and $50,000 \mathrm{~K}$ for the temperature, taken from a representative case of $100 \mathrm{eV}$ ions at normal incidence (the corresponding Maxwellian VDF is plotted alongside the Sigmund-Thompson distribution described above in Fig. 5). The present work assesses the validity of that assumption via a parametric, computational study, wherein the bulk velocity and temperature of the sputtered boron atoms are varied and qualitative changes in the boron density downstream are analyzed.

\section{B. Hall Thruster Simulation Setup}

The Hall thruster modeled in the present work is NASA's High-Voltage Hall Accelerator, or HiVHAc. ${ }^{15-18}$ The HiVHAc project is jointly conducted by NASA Glenn Research Center and Aerojet Rocketdyne with the goal of producing a long-life, high-specific-impulse Hall thruster for Discovery-class missions. The current engineering development unit (EDU2), has demonstrated operation at discharge voltages up to $650 \mathrm{~V}$ and discharge powers in excess of $4 \mathrm{~kW}$.

The operating conditions simulated in the present work are given in Table 1. These particular operating points are chosen because they have been investigated experimentally using cavity ringdown spectroscopy (CRDS), a technique for measuring the density of a target species integrated along a laser path. ${ }^{19}$ Lee et al. utilized CRDS to determine the density of ground-state boron atoms in the HiVHAc plume. ${ }^{20}$ In our previous work, results from HPHall-3 showed reasonably good agreement with Lee's CRDS measurements considering the numerous uncertainties and assumptions associated with the boron submodel and HPHall-3 in gen-

Table 1: Discharge voltage, propellant mass flow rate, and discharge current for the operating points studied in the boron transport simulations.

\begin{tabular}{ccccc}
\hline \multirow{2}{*}{$V_{d}, \mathrm{~V}$} & $\dot{m}_{a}, \mathrm{mg} / \mathrm{s}$ & \multicolumn{3}{c}{$I_{d}, \mathrm{~A}$} \\
& & Nominal & Exp. & Sim. \\
\hline 500.5 & 2.36 & 2 & 1.99 & 2.00 \\
500.1 & 4.26 & 4 & 3.94 & 4.02 \\
500.6 & 6.04 & 6 & 6.04 & 6.08 \\
\hline
\end{tabular}
eral. ${ }^{2,3}$ In the present work, Lee's measurements serve as the benchmark against which the results of the parametric study are assessed.

All simulations are performed on a $70 \times 30$ cell elliptical mesh, shown in Fig. 6 . The base time step is $50 \mathrm{~ns}$, and the electron time step is 1/1250 of the base time step. Simulations progress as follows: First, the 


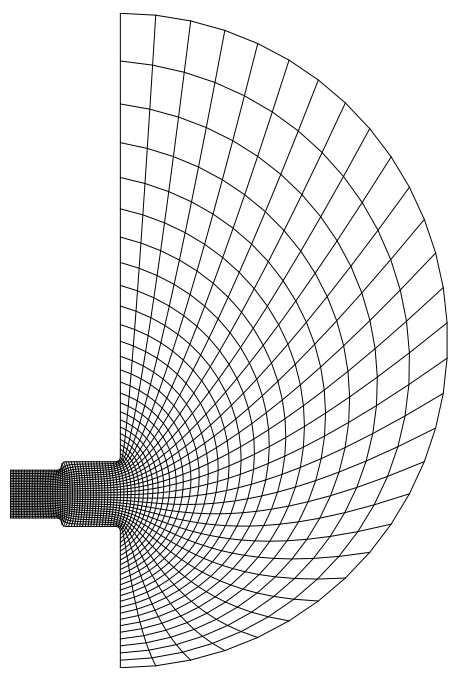

Figure 6: Elliptical mesh used in HPHall-3 simulations.

domain is populated with neutral macroparticles over 20,000 time steps. Then, the plasma physics are turned on and the simulation runs for another 5000 time steps to allow any startup transients to stabilize. Another 40,000 time steps are then processed to calculate the steady-state performance parameters for comparison to measured performance data. Finally, the boron submodel is turned on and the simulation is run for another 20,000 time steps before calculating the time-averaged boron density. The input parameters varied between simulations are the Maxwellian mean velocity along the direction of forward sputtering and the temperature of the Maxwellian along the directions of forward and transverse sputtering. The values chosen for these parameters are based on the observed variations in the MD data. ${ }^{3,6}$ Any elastic scattering collisions involving boron atoms are neglected due to their long mean free path compared to the thruster dimensions.

\section{Simulation Results}

\section{A. Baseline Plasma Properties}

Figure 7 shows contours of plasma density and electron temperature in HiVHAc operating at $500 \mathrm{~V}$ and 4 A. These contours are provided to give context to the discussion of the boron density below. From Fig. 7, we see that the maximum plasma density is about $2.3 \times 10^{18} \mathrm{~m}^{-3}$ at around $z / L=-0.4$. The exact value of the plasma density will vary between operating points, but the qualitative behavior is characteristic of all operating points investigated. The electron temperature, meanwhile, reaches a peak of about $48 \mathrm{eV}$ just upstream of the channel exit, around $z / L=-0.1$. Again, exact values vary, but the behavior is typical of all operating points.

We can make a few predictions based on Fig. 7 alone. First, boron atoms should originate from the walls where the electron temperature is greatest. This region corresponds to the thruster acceleration zone and to relatively large wall sheath potentials, so there are proportionally more energetic ions striking the walls in this region, which in turn means more boron atoms are sputtered. Second, the peak boron density should be on the order of $10^{17} \mathrm{~m}^{-3}$ or less, and is likely an order of magnitude or more smaller than that. This is because only a fraction of ions strike the walls, and not all ion impacts cause sputtering events. Hence, the boron density should be much less than the plasma density. Indeed, the predicted behavior in the boron density was observed in past work. ${ }^{2,3}$

\section{B. Boron Density Contours}

Figure 8 shows contours of ground-state boron number density for the case of $500 \mathrm{~V}$ discharge voltage and $2 \mathrm{~A}$ discharge current. Figure 8a corresponds to the baseline values for the Maxwellian mean velocity and temperature. As the contours show, introducing a nonzero mean velocity along the forward sputtering direction cause the boron to accumulate further downstream. This is because most of the ions striking the 


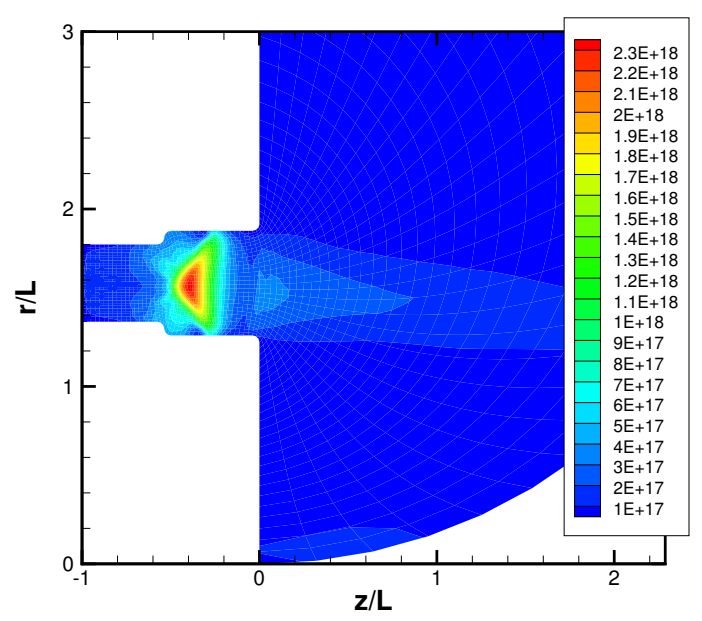

(a) Plasma density $\left(\mathrm{m}^{-3}\right)$

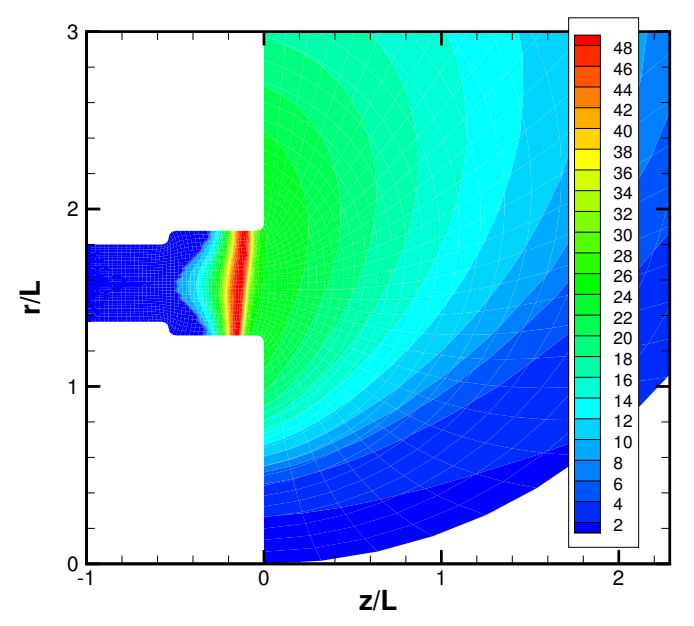

(b) Electron temperature (eV)

Figure 7: Plasma density and electron temperature in the HiVHAc thruster operating at $500 \mathrm{~V}$ and $4 \mathrm{~A}$.

walls are moving axially downstream along the electric field, so sputtered atoms are more likely to move in that same direction. As the mean velocity $\bar{v}_{x}$ is increased in magnitude the effects become more pronounced, with an obvious outward bulge in the boron density contours appearing for $\bar{v}_{x}=3000 \mathrm{~m} / \mathrm{s}$. Increasing the temperature has a more subtle effect, causing sputtered boron atoms to diffuse more rapidly in all directions. This results in a slightly more uniform distribution of boron atoms in the discharge channel and a lower boron density far downstream of the thruster (i.e., $z / L>1$ ).

Curiously, Fig. 8 seems to indicate that increasing the Maxwellian mean velocity along the forward sputtering direction results in increased boron density throughout the thruster plume. There are two effects at work here: First, as more boron atoms are directed downstream, fewer of those atoms are "lost" through redeposition on the walls and more atoms escape into the plume. Second, on average, increasing the mean velocity results in a corresponding decrease in the angle between a sputtered atom's velocity vector and the downstream axis, so fewer atoms should be found near the channel exit plane at extreme values of $r / L$. However, because the thermal velocity of the sputtered atoms is about much greater than the mean velocity, the first effect dominates and more boron is seen everywhere in the thruster plume.

Figure 9 shows contours of ground-state boron density for HiVHAc operating at $500 \mathrm{~V}$ and $6 \mathrm{~A}$. Due to the increased propellant flow, the boron number density is increased compared to the $2 \mathrm{~A}$ operating point, but the trends with the boron VDF parameters are the same: Increasing the bulk velocity of boron atoms causes the contours to shift axially downstream and increases the density of boron throughout the plume region, whereas increasing the temperature of the boron atoms causes the boron atoms to be distributed more uniformly in the discharge channel, with fewer boron atoms in the plume. In other words, the behavior of the sputtered boron atoms is more or less unchanged by the increase in discharge current, but the amount of atoms sputtered is increased, as expected.

\section{CRDS Comparison}

Figure 10 shows the time-averaged boron number density and path-integrated number density calculated from the HPHall-3 data and measured by Lee et al. for the $2 \mathrm{~A}, 4 \mathrm{~A}$, and $6 \mathrm{~A}$ operating points. ${ }^{20}$ The nondimensional beam position and thruster radius are both defined as

$$
R=\frac{r-r_{\text {inner }}}{r_{\text {outer }}-r_{\text {inner }}}
$$

where $r_{\text {inner }}$ and $r_{\text {outer }}$ are the inner and outer channel wall radius, respectively, so $R=0$ corresponds to the 


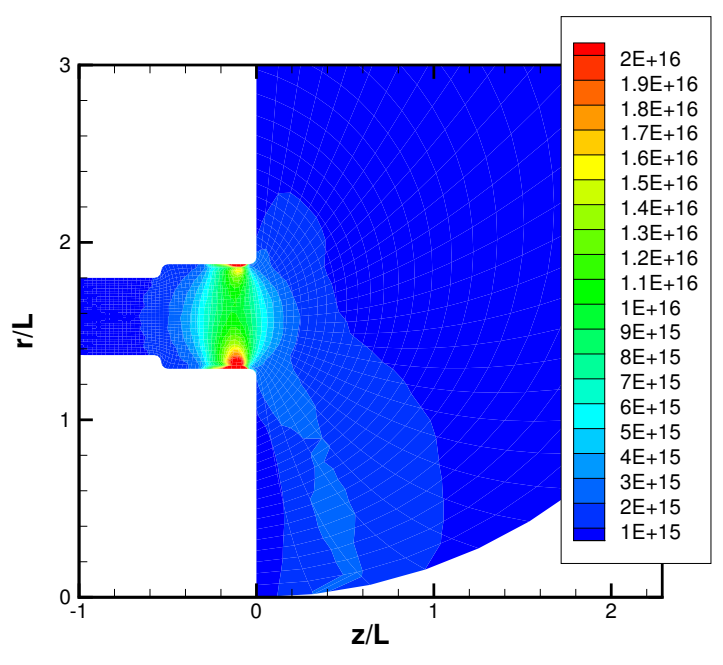

(a) $\bar{v}_{x}=0, T_{x y}=50,000 \mathbf{K}$

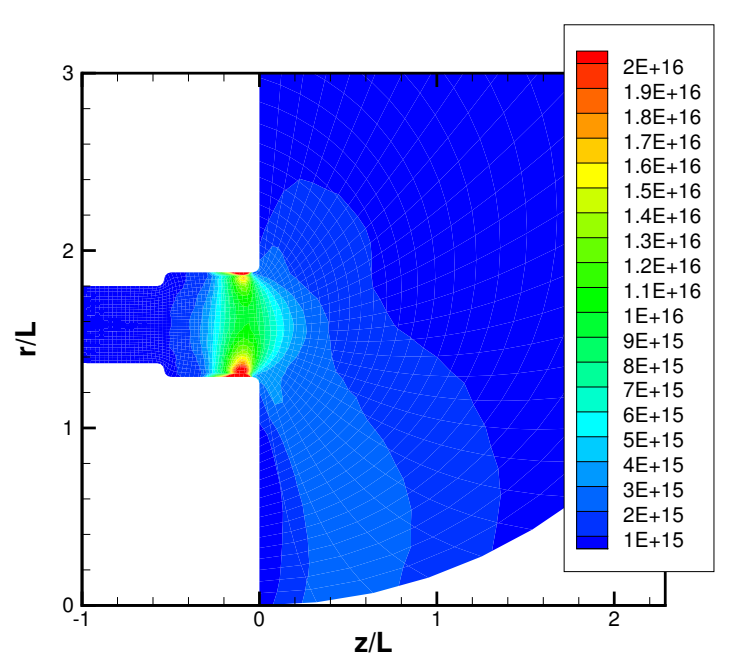

(c) $\bar{v}_{x}=3000 \mathbf{m} / \mathbf{s}, T_{x y}=50,000 \mathbf{K}$

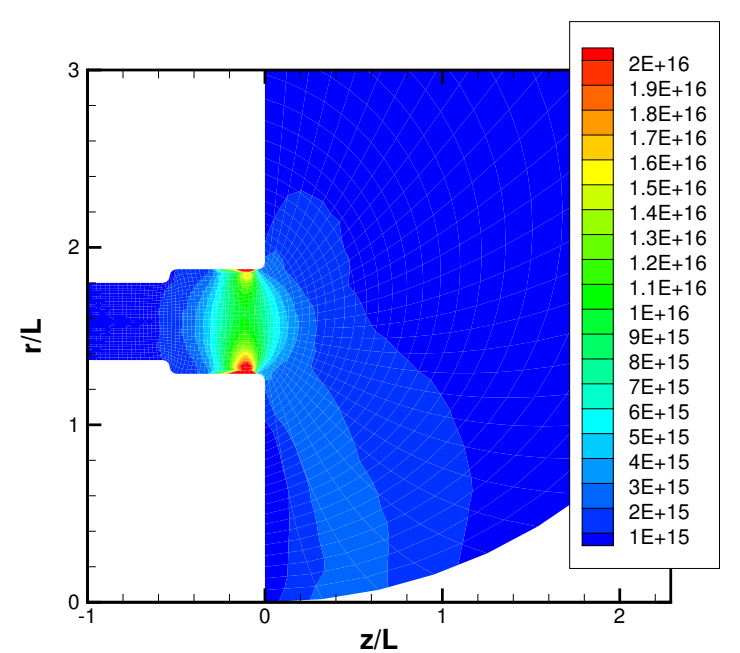

(b) $\bar{v}_{x}=1000 \mathrm{~m} / \mathrm{s}, T_{x y}=50,000 \mathbf{K}$

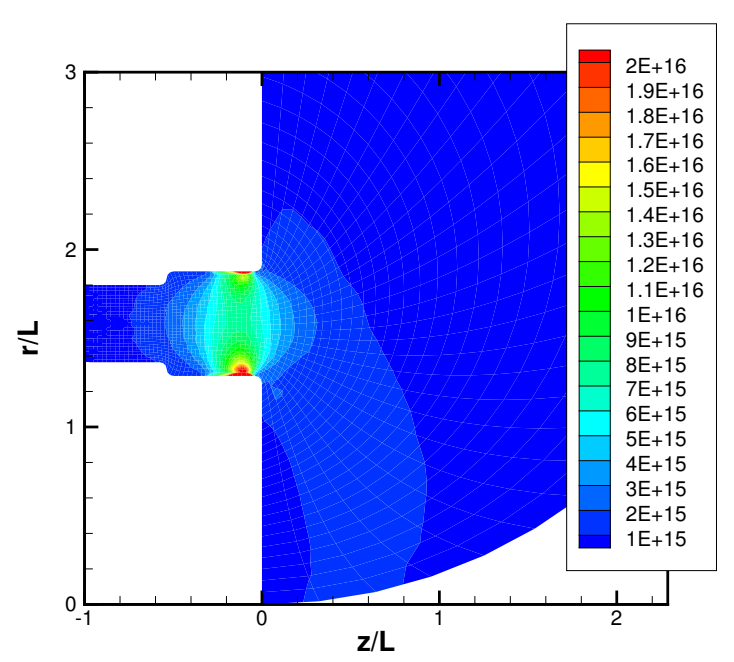

(d) $\bar{v}_{x}=0, T_{x y}=100,000 \mathbf{K}$

Figure 8: Number density of ground-state boron atoms $\left(\mathrm{m}^{-3}\right)$ in HiVHAc operating at $500 \mathrm{~V}$ and $2 \mathrm{~A}$. 


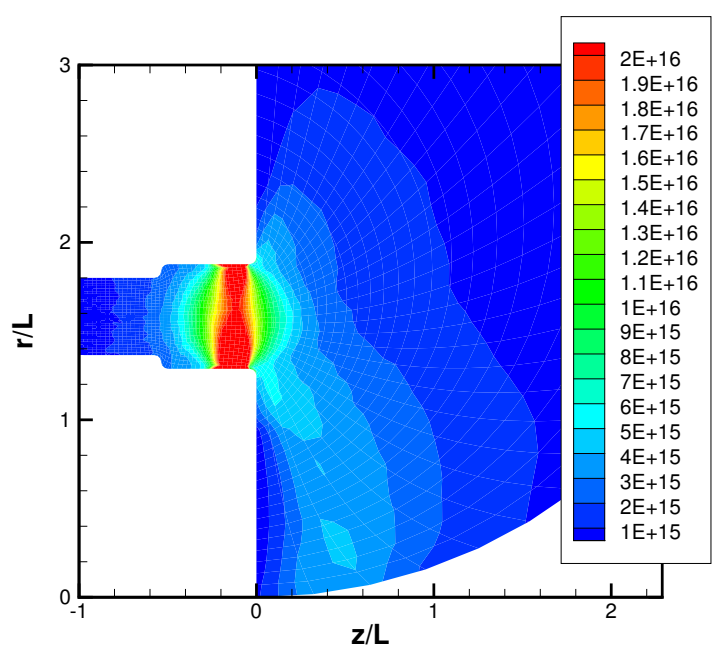

(a) $\bar{v}_{x}=0, T_{x y}=50,000 \mathbf{K}$

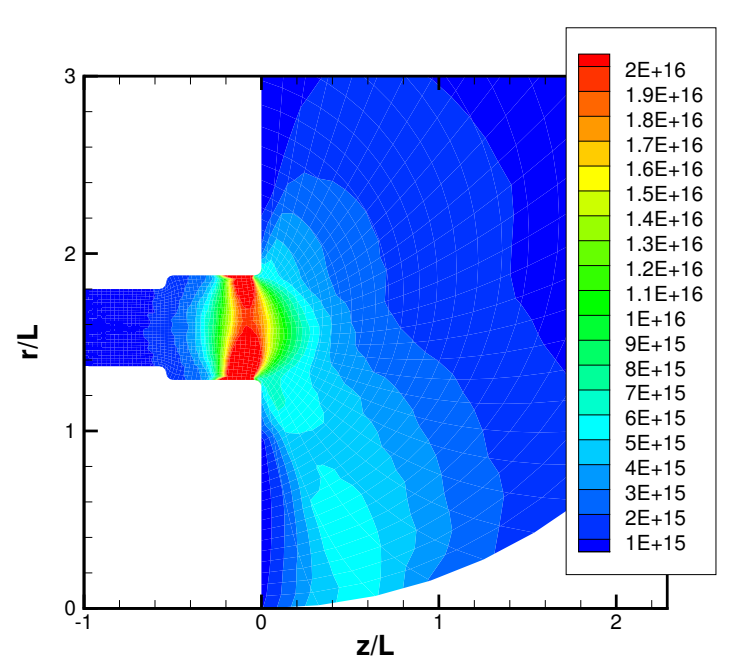

(c) $\bar{v}_{x}=3000 \mathbf{m} / \mathbf{s}, T_{x y}=50,000 \mathbf{K}$

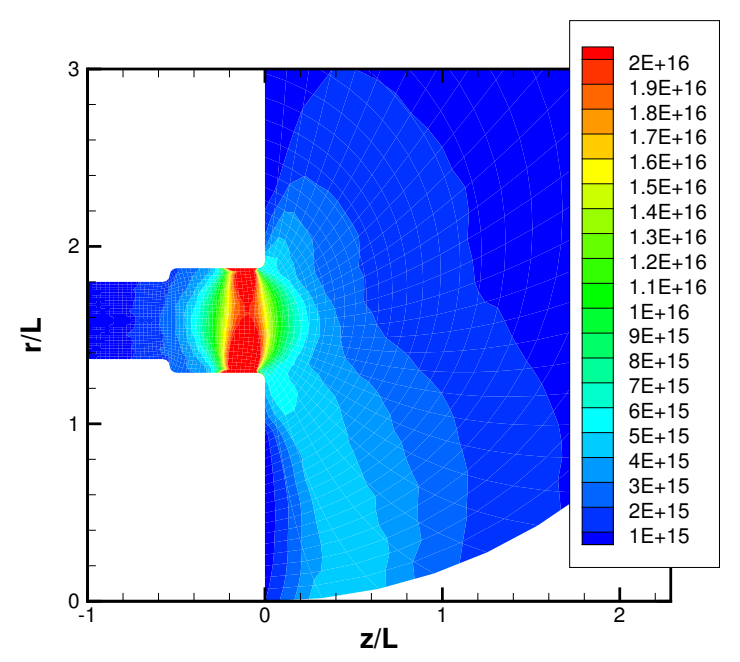

(b) $\bar{v}_{x}=1000 \mathrm{~m} / \mathrm{s}, T_{x y}=50,000 \mathbf{K}$

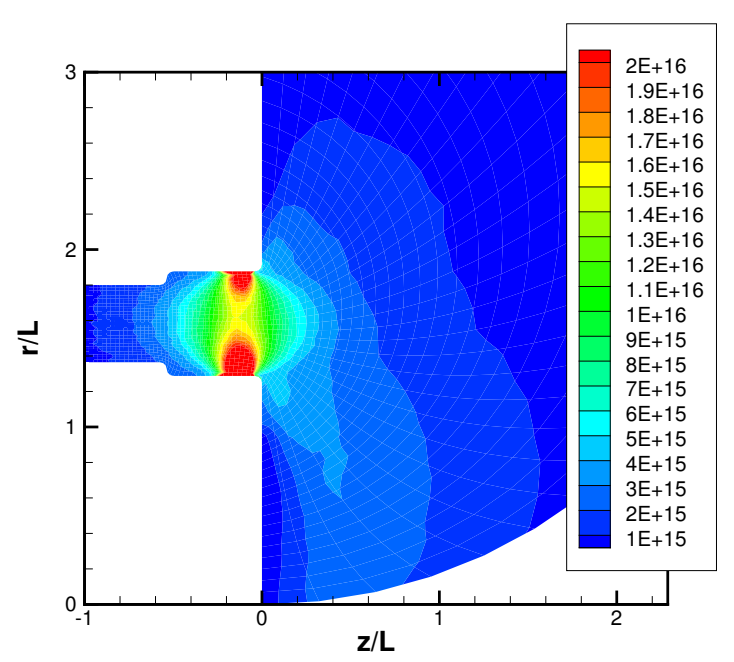

(d) $\bar{v}_{x}=0, T_{x y}=100,000 \mathbf{K}$

Figure 9: Number density of ground-state boron atoms $\left(\mathrm{m}^{-3}\right)$ in HiVHAc operating at $500 \mathrm{~V}$ and $6 \mathrm{~A}$. 


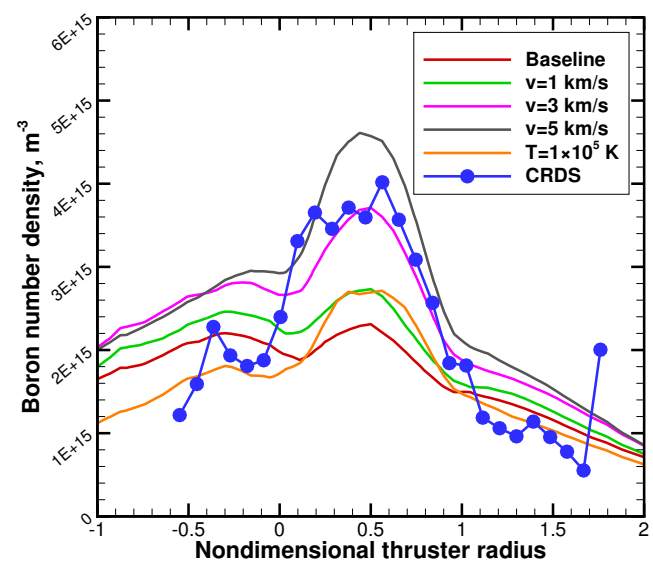

(a) $500 \mathrm{~V}, 2 \mathrm{~A}$

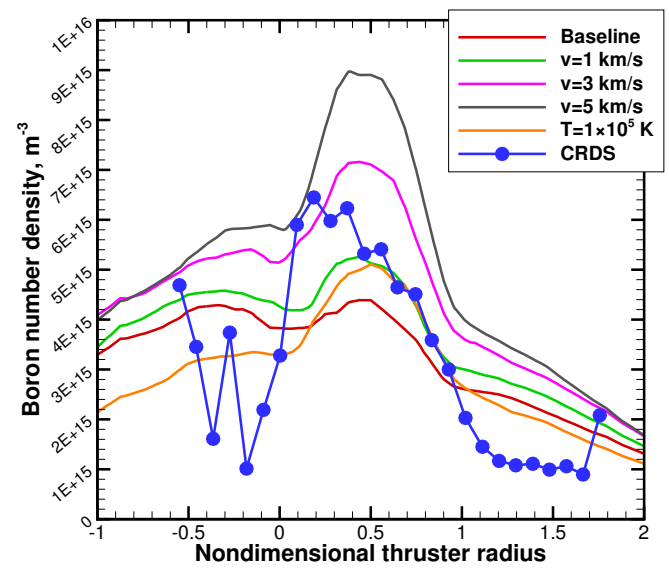

(c) $500 \mathrm{~V}, 4 \mathrm{~A}$

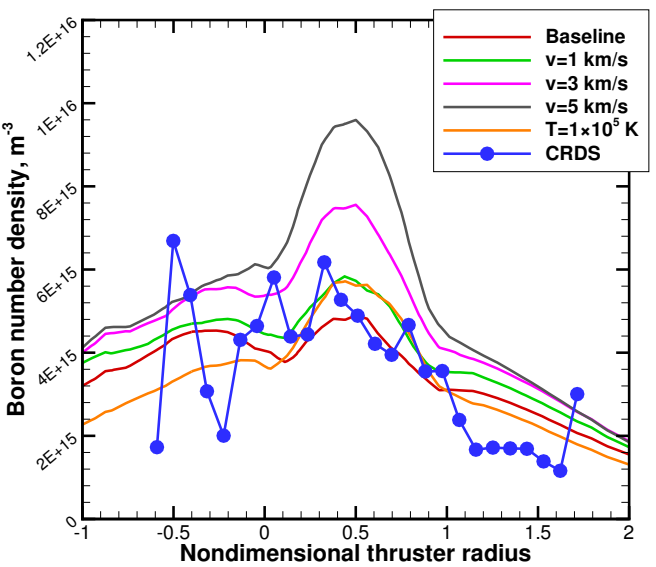

(e) $500 \mathrm{~V}, 6 \mathrm{~A}$

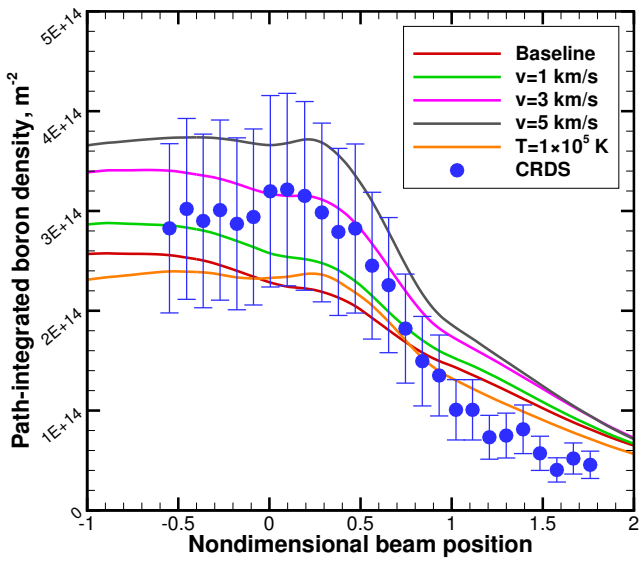

(b) $500 \mathrm{~V}, 2 \mathrm{~A}$

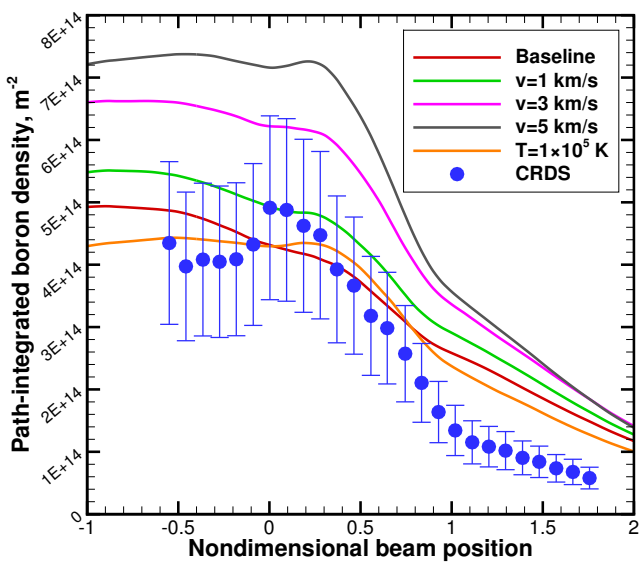

(d) $500 \mathrm{~V}, 4 \mathrm{~A}$

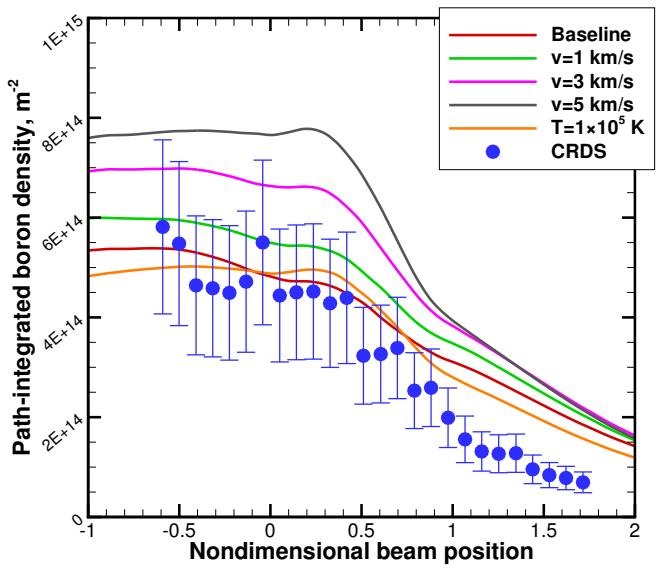

(f) $500 \mathrm{~V}, 6 \mathrm{~A}$

Figure 10: Ground-state boron number density (left) and path-integrated number density (right) $6 \mathrm{~mm}$ downstream of the HiVHAc channel exit plane as calculated from HPHall-3 and measured using CRDS. 
inner wall and $R=1$ corresponds to the outer wall. The error bars on the CRDS measurements correspond to an estimated uncertainty of $30 \%$.

Just as with Figs. 8 and 9, Fig. 10 indicates that an increase in the Maxwellian mean velocity along the direction of forward sputtering causes an increase in the boron density throughout the plume. However, this increase in boron density is most prominent in the region radially between the inner and outer walls of the thruster, i.e. $0<R<1$. This corresponds to the outward bulge observed in the boron density contours at larger values of $\bar{v}_{x}$ and falls within expectations. The noticeably increased boron density in the region of $0<R<1$ falls in line with the deconvolved CRDS data, which indicate a peak in the boron density in that region. The results vary between operating conditions, but it appears that a bulk velocity of $1000-3000 \mathrm{~m} / \mathrm{s}$ results in the best agreement between the simulation results and experimental measurements in that region. However, for $R<0$ and $R>1$, the increased boron density at high mean velocities results in poor relatively poor agreement with the experimental data.

Doubling the boron temperature from $50,000 \mathrm{~K}$ to $100,000 \mathrm{~K}$ has a much more interesting effect on the boron density profiles. Namely, the boron density in the region of $0<R<1$ is noticeably increased, but the boron density outside this region is decreased. This results in a qualitative profile that more closely resembles the CRDS measurements, with a very low boron density at large positive $R$, a peak in the region of $0<R<1$, and lower density again for $R<0$. Hence, the temperature of the sputtered boron atoms seems to have a more significant effect on the radial distribution of boron atoms just downstream of the thruster exit than the mean velocity, whereas the mean velocity largely determines the amount of boron that escapes into the plume.

The above observations indicate that both the mean velocity and temperature of sputtered boron atoms along the forward and transverse sputtering directions have a very significant effect on the distribution of boron atoms downstream. Given this fact, future studies of boron transport in Hall thrusters must account for the dependence of the bulk velocity and temperature of sputtered boron atoms on the properties of ions striking the discharge channel walls. This is particularly important when investigating the problem of spacecraft integration, as boron is a condensible species and could deposit on optical instruments or solar panels, thus jeopardizing a mission.

\section{Conclusions and Future Work}

In this work, a parametric study was performed to analyze the effects of boron velocity distribution functions (VDFs) on the transport and distribution of boron atoms in a Hall thruster. Using data from molecular dynamics (MD) simulations and assuming a Maxwellian velocity distribution in the directions of forward and transverse sputtering, several simulations of NASA's HiVHAc Hall thruster were performed using the hybrid fluid/particle-in-cell model HPHall with various values for the bulk velocity of boron atoms in the forward sputtering direction and the temperature of sputtered atoms in the forward and transverse sputtering directions. A Sigmund-Thompson velocity distribution with fixed parameters was used for the wall normal direction. It was found that increasing the bulk velocity of the sputtered boron atoms caused an increase in boron density throughout the thruster plume, especially in the region lying radially between the inner and outer channel walls of the thruster. Increasing the temperature of the sputtered atoms resulted in a more uniform distribution of boron atoms in the discharge channel and a decreased density of boron atoms deep in the plume. When compared to experimental measurements of boron density using CRDS, it was found that a moderate bulk velocity of $1000-3000 \mathrm{~m} / \mathrm{s}$ resulted in significantly better agreement between the simulation results and experimental measurements in the region radially between the inner and outer channel walls of the thruster. At more extreme radial positions, the simulations using a positive bulk velocity show that the boron density is greater than observed experimentally. Conversely, increasing the temperature of the sputtered boron atoms resulted in better qualitative agreement between the calculated and measured boron density profiles. These observations indicate that the bulk velocity of the boron atoms largely controls the amount of boron that escapes into the thruster plume, whereas the temperature of the boron atoms determines how that boron is distributed in the plume.

This study focused on the distribution of sputtered boron atoms in the near-field plume of a thruster simply because experimental data were available for comparison. For spacecraft integration purposes, the distribution of boron farther away from the thruster is likely more important. Hence, a simulation including the far-field plume could be performed to determine the sensitivity of boron density in that region to the VDF parameters used to inject boron atoms into the simulation. If higher resolution of the boron VDFs is 
required, empirical curve fits to the VDF parameters calculated from the MD model could be performed, thus quantifying the dependence on ion energy and incidence angle. Then, the boron submodel could be altered so that sputtered boron atoms are injected into the domain with the correct velocity distribution based on the energy and incidence angle of the associated ion.

\section{References}

${ }^{1}$ Fife, J., Hybrid-PIC Modeling and Electrostatic Probe Survey of Hall Thrusters, Ph.D. thesis, Massachusetts Institute of Technology, 1999.

${ }^{2}$ Smith, B. D., Boyd, I. D., and Kamhawi, H., "Hybrid-PIC Modeling of the Transport of Atomic Boron in a Hall Thruster," 34th International Electric Propulsion Conference, IEPC Paper 2015-252, Kobe, Japan, July 2015.

${ }^{3}$ Smith, B. D., A Numerical Study of Wall Erosion and Material Transport in Hall Thrusters, Ph.D. thesis, University of Michigan, 2015.

${ }^{4}$ Smith, B. D. and Boyd, I. D., "Computation of Total and Differential Sputter Yields of Boron Nitride Using Molecular Dynamics," 33rd International Electric Propulsion Conference, IEPC Paper 2013-156, Washington, DC, USA, Oct. 2013.

${ }^{5}$ Smith, B. D. and Boyd, I. D., "Molecular Dynamics Computation of Steady-State Sputter Yields of Hexagonal Boron Nitride," 34th International Electric Propulsion Conference, IEPC Paper 2015-278, Kobe, Japan, July 2015.

${ }^{6}$ Smith, B. D. and Boyd, I. D., "Molecular dynamics investigation of hexagonal boron nitride sputtering and sputtered particle characteristics," Journal of Applied Physics, submitted April 2016.

${ }^{7}$ Huismann, T., Improving Hall Thruster Plume Simulation through Refined Characterization of Near-field Plasma Properties, Ph.D. thesis, University of Michigan, 2011.

${ }^{8}$ Smith, B. D., Boyd, I. D., and Kamhawi, H., "Influence of Triply-Charged Ions and Ionization Cross-Sections in a Hybrid-PIC Model of a Hall Thruster Discharge," 50th AIAA/ASME/SAE/ASEE Joint Propulsion Conference, AIAA Paper 2014-3429, Cleveland, Ohio, USA, July 2014, pp. 1-17.

- ${ }^{9}$ Bohdansky, J., "A universal relation for the sputtering yield of monatomic solids at normal ion incidence," Nuclear Instruments and Methods in Physics Research B, Vol. 2, No. 1-3, 1984, pp. 587-591.

${ }^{10}$ García-Rosales, C., Eckstein, W., and Roth, J., "Revised formulae for sputtering data," Journal of Nuclear Materials, Vol. 218, No. 1, 1995, pp. $8-17$.

$\checkmark{ }^{11}$ Biersack, J. and Ziegler, J., "The Stopping and Range of Ions in Solids," Ion Implantation Techniques, edited by H. Ryssel and H. Glawischnig, Vol. 10 of Springer Series in Electrophysics, Springer Berlin Heidelberg, 1982, pp. 122-156.

$\checkmark{ }^{12}$ O'connor, D. and Biersack, J., "Comparison of theoretical and empirical interatomic potentials," Nuclear Instruments and Methods in Physics Research B, Vol. 15, 1986, pp. 14-19.

13Yamamura, Y. and Tawara, H., "Energy Dependence of Ion-Induced Sputtering Yields From Monatomic Solids At Normal Incidence," Atomic Data and Nuclear Data Tables, Vol. 62, No. 2, 1996, pp. 149-253.

${ }^{14}$ Sigmund, P., "Theory of Sputtering. I. Sputtering Yield of Amorphous and Polycrystalline Targets," Physical Review, Vol. 184, No. 2, 1969, pp. 383-416.

${ }^{15}$ Kamhawi, H., Manzella, D., Pinero, L., Haag, T., Mathers, A., and Liles, H., "In-Space Propulsion High Voltage Hall Accelerator Development Project Overview," 45th AIAA/ASME/SAE/ASEE Joint Propulsion Conference \& Exhibit, AIAA Paper 2009-5282, Denver, Colorado, USA, Aug. 2009, pp. 1-12.

${ }^{16}$ Kamhawi, H., Manzella, D., Pinero, L., Haag, T., and Huang, W., "In-Space Propulsion High Voltage Hall Accelerator Development Project Overview," 46th AIAA/ASME/SAE/ASEE Joint Propulsion Conference Es Exhibit, AIAA Paper 20106860, Nashville, Tennessee, USA, July 2010.

${ }^{17}$ Kamhawi, H., Haag, T., Pinero, L., Huang, W., Peterson, T., Manzella, D., Dankanich, J., Mathers, A., and Hobson, D., "Overview of the Development of a Low-Cost High Voltage Hall Accelerator Propulsion System for NASA Science Missions," 47th AIAA/ASME/SAE/ASEE Joint Propulsion Conference \& Exhibit, No. August in AIAA Paper 2011-5520, San Diego, California, USA, 2011, pp. 1-11.

${ }^{18}$ Kamhawi, H., Pinero, L., Haag, T., Peterson, T., Huang, W., Dankanich, J., Shastry, R., and Mathers, A., "High Voltage Hall Accelerator Propulsion System Development for NASA Science Missions," 2013 IEEE Aerospace Conference, Big Sky, Montana, USA, March 2013.

${ }^{19}$ Huang, W., Study of Hall Thruster Discharge Channel Wall Erosion via Optical Diagnostics by Wensheng Huang, Ph.D. thesis, University of Michigan, 2011.

${ }^{20}$ Lee, B. C., Yalin, A. P., Gallimore, A., Huang, W., and Kamhawi, H., "Real-Time Boron Nitride Erosion Measurements of the HiVHAc Thruster via Cavity Ring-Down Spectroscopy," 33rd International Electric Propulsion Conference, IEPC Paper 2013-119, Washington, DC, USA, Oct. 2013. 